\title{
Psychometric evaluation of the Snaith-Hamilton Pleasure Scale (SHAPS) in adult outpatients with major depressive disorder
}

\author{
Paul A. Nakonezny ${ }^{a}{ }^{*}$, Thomas J. Carmodya ${ }^{a}$, David W. Morris ${ }^{b}$, Benji T. Kurian ${ }^{b}$, and \\ Madhukar H. Trivedib \\ aDepartment of Clinical Sciences, Division of Biostatistics, The University of Texas Southwestern \\ Medical Center, 6363 Forest Park Rd, Suite 651, Dallas, TX 75390-8828, USA \\ ${ }^{b}$ Department of Psychiatry, The University of Texas Southwestern Medical Center, 5323 Harry \\ Hines Blvd, Dallas, TX 75390-9119, USA
}

\section{Abstract}

The inability to experience pleasure, anhedonia, is recognized as a hallmark symptom of depression. An instrument developed for the assessment of hedonic capacity is the 14-item, self-report, SnaithHamilton Pleasure Scale, but its psychometric properties have not been adequately evaluated. The current study examined the reliability and validity of the SHAPS using a large sample of adult outpatients with major depressive disorder (MDD). Data for the current study were obtained from 461 adult outpatients with a diagnosis of MDD who participated in Project IMPACTS. Internal consistency of the SHAPS was assessed using Cronbach's coefficient alpha. A Principal Factor Analysis was used to define the dimensionality of the SHAPS. Convergent and discriminant validity was assessed by evaluating the Pearson correlations between the SHAPS total score and the pleasure/ enjoyment item of the IDS-C 30 , QLES-Q, HRSD 17 , IDS-C ${ }_{30}$, QIDS-C ${ }_{16}$, and MADRS M $_{10}$, respectively. The internal consistency of the SHAPS was .91. A one-factor solution emerged for the SHAPS (eigenvalues of the first two initial factors were 5.95 and 0.43 , respectively). Pearson correlations revealed a positive linear relationship between the SHAPS total score and the total scores on the $\operatorname{HRSD}_{17}(r=.49, p<.0001)$, IDS-C $30(r=.56, p<.0001)$, QIDS- $\mathrm{C}_{16}(r=.55, p<.0001)$, and MADRS $_{10}(r=.53, p<.0001)$. The SHAPS total score was negatively correlated with the QLES-Q $(r=-0.65, \mathrm{p}<.0001)$. The current study shows that the SHAPS is a reliable, valid, and unidimensional instrument to assess hedonic capacity in adult outpatients with MDD.

\section{Keywords}

Major depression; hedonic capacity; Snaith-Hamilton pleasure scale; reliability and validity

\section{Introduction}

Anhedonia, the inability to experience pleasure, is recognized as a hallmark symptom of depression, and is included as a primary symptom in the diagnostic criteria for clinical depression in both the Diagnostic and Statistical Manual of Mental Disorders Fourth Edition (DSM IV; APA, 2000) and the International Statistical Classification of Diseases and Related

"Corresponding author. Department of Clinical Sciences, Division of Biostatistics, The University of Texas Southwestern Medical Center, 6363 Forest Park Rd, Suite 651, Dallas, TX 75390-8828, USA. Tel: 214-648-5289, Fax: 214-648-5323.

paul.nakonezny@utsouthwestern.edu.

Declaration of interest statement The authors have no conflict of interest that could have inappropriately influenced, or be perceived to influence, this work. 
Health Problems (ICD; WHO, 1992). Although low hedonic capacity or anhedonia is considered a core symptom in the psychopathology of major depressive disorder, its measurement in depression has received little research attention. In fact, measures of hedonic capacity have been excluded from most studies in favor of multiple measures of depression severity. An instrument developed for the assessment of hedonic capacity is the 14-item, selfreport, Snaith-Hamilton Pleasure Scale (SHAPS; Snaith et al., 1995). The SHAPS was developed to minimize cultural, gender, and age biases in the evaluation of hedonic capacity (Snaith et al., 1995), and has potential for use in both clinical and research settings, but the validity and reliability of the SHAPS have not been adequately established in MDD patients. The three validation studies to our knowledge that examined the psychometric properties of the SHAPS in a clinical setting used the French (Loas et al., 1997), German (Franz et al., 1998), or Dutch (Franken et al., 2007) version of the SHAPS and used small clinical samples of MDD or schizophrenic inpatients (sample sizes ranged from 20 to 103 inpatients). And there have been no studies (to our knowledge) that have assessed the psychometric properties of the SHAPS (English or foreign versions) in adult outpatients with major depressive disorder.

The purpose of the current study was to examine the psychometric properties of the original (English) version of the SHAPS (Snaith et al., 1995) using a large generalizable sample of 461 adult outpatients with MDD. Using classical test theory analysis, we examined the internal consistency, scale dimensionality, and convergent and discriminant validity of the SHAPS. Also, in this study, we examined the relationship of the SHAPS scores with participant demographic and clinical characteristics.

\section{Methods}

\section{Study and Participants}

Data were obtained from Project IMPACTS (Implementation of Algorithms using Computerized Treatment Systems). The procedures and methods of Project IMPACTS have been detailed elsewhere (Trivedi et al., 2007). Briefly, Project IMPACTS examined the effectiveness of a computer-based decision support system for algorithm-based treatment of major depressive disorder across four domains: patient outcomes, direct cost of treatment, impact on physicians' behavior and attitudes, and overall health care cost versus outcome (Trivedi et al., 2007). The IMPACTS patient outcome data (study exit or the date closest to one year), which include symptom severity, hedonic capacity, social and work function, and quality of life, were used in the current paper. Patients with no postbaseline data were excluded. Participants for the current study were 461 adult outpatients (18 years of age and older) with a diagnosis of MDD (with or without psychotic symptoms), as verified by the Structured Clinical Interview for DSM-IV (SCID) (First et al., 1995) and a 17-item Hamilton Rating Scale for Depression $\left(\mathrm{HRSD}_{17}\right)$ score of 14 or greater (Hamilton, 1960) that were treated by a studyenrolled clinician.

\section{Measures}

The current study used four separate clinician-rated instruments to measure depressive severity, a self-administered instrument to measure hedonic capacity, and a self-administered instrument to measure quality of life, enjoyment, and satisfaction. The clinician raters of depressive severity were blinded to treatment assignment.

Measure of Hedonic Capacity-The 14-item Snaith-Hamilton Pleasure Scale (SHAPS; Snaith, 1993). The SHAPS is a self-administered instrument that was used to measure hedonic capacity (Snaith, 1993). The SHAPS items are displayed in Table 1. Each of the items has a set of four response categories--Definitely Agree, Agree, Disagree, and Strongly Disagree, with either of the Disagree responses receiving a score of 1 and either of the Agree responses 
receiving a score of 0 . Thus, the SHAPS was scored as the sum of the 14 items so that total scores ranged from 0 to 14 . A higher total SHAPS score indicated higher levels of present state of anhedonia.

Measure of Quality of Life, Enjoyment, and Satisfaction-Quality of Life, Enjoyment, and Satisfaction Questionnaire (QLES-Q short form; Endicott et al., 1993). The QLES-Q Short Form is a 16-item self-administered questionnaire designed to measure degree of enjoyment and satisfaction in general activities. The QLES-Q measured each of the items using a 5-point Likert-type scale. The QLES-Q was first scored as the sum of the first 14 items so that total scores ranged from 14 to 70 and then the sum was converted into a percentage of the maximum possible score. Higher QLES-Q scores reflected greater enjoyment and satisfaction with activities. The QLES-Q has previously been found to have acceptable reliability and validity (Endicott, 1993).

Measures of Depression Severity-The 17-item Hamilton Rating Scale for Depression (HRSD $_{17}$; Hamilton, 1960). The $\mathrm{HRSD}_{17}$ is a widely used clinician-administered rating scale that is designed to assess symptom severity in patients diagnosed with depression (Schwab et al., 1967). Total score on the $\mathrm{HRSD}_{17}$ ranges from 0 to 52, with higher scores representing greater severity of depressive symptoms. The $\mathrm{HRSD}_{17}$ has established psychometric properties (Hamilton, 1960; Schwab et al., 1967).

The 16-item Quick Inventory of Depressive Symptomatology (QIDS-C ${ }_{16}$; Rush et al., 2003; Trivedi et al., 2004). The QIDS- $\mathrm{C}_{16}$, the clinician-administered version, assesses the nine symptom domains which constitute the DSM-IV criteria for depression: sad mood, lack of interest, feelings of guilt, suicidal ideation, decreased energy, sleep disturbance, appetite/ weight increase/decrease, and psychomotor agitation/retardation. Total score on the QIDS$\mathrm{C}_{16}$ ranges from 0 to 27 , with higher scores representing greater severity of depressive symptoms. The psychometric properties of the QIDS- $\mathrm{C}_{16}$ have been extensively evaluated (Rush et al., 2003, 1996; Trivedi et al., 2004).

The 30-item Inventory of Depressive Symptomatology - Clinician-rating (IDS-C ${ }_{30}$; Rush et al., 1996; Trivedi et al., 2004). The IDS- $\mathrm{C}_{30}$ includes the 16 items contained on the QIDS$\mathrm{C}_{16}$, as well as 14 additional items designed to assess melancholic, atypical, and anxious features. Total score on the IDS- $\mathrm{C}_{30}$ ranges from 0 to 84 , with higher scores representing greater severity of depressive symptoms. The pleasure/enjoyment item of IDS- $\mathrm{C}_{30}$ (item 21) was also used to evaluate convergent validity of the SHAPS. The pleasure/enjoyment item of the IDS$\mathrm{C}_{30}$ was scored using a 4-point scale that ranged from 0 (derives usual sense of enjoyment from pleasurable activities) to 3 (unable to register any sense of pleasure/enjoyment from anything). The psychometric properties of the IDS- $\mathrm{C}_{30}$ have been previously evaluated (Rush et al., 2003, 1996; Trivedi et al., 2004).

The 10-item clinician-rated Montgomery-Asberg Depression Rating Scale (MADRS 10 ; Montgomery and Äsberg, 1979). The MADRS 10 is one of the most widely used clinician-rated assessments of depressive severity, with well-established psychometric properties (Khan et al., 2002; Galinowski and Lehert, 1995). Total score on the MADRS 10 ranges from 0 to 60, with higher scores representing greater severity of depressive symptoms.

\section{Statistical Analysis}

The Pearson/point-biserial partial correlation coefficient (controlling for QIDS- $_{16}$ at exit) was used to assess the linear relationship between SHAPS total score (at exit) and participant demographic and clinical characteristics. For the point-biserial correlation analysis, gender, race, and first versus recurrent episode of depression were binary indicators; gender coded as 
male $=0$ and female $=1$, race coded as Caucasian $=0$ and non-Caucasian $=1$, and first versus recurrent episode of depression coded as first $=0$ and recurrent $=1$.

Classical test theory (CTT) analysis was used to generate the mean, standard deviation, and item/total correlation $\left(r_{i t}\right)$ for each SHAPS item as well as the overall scale mean and scale standard deviation. CTT analysis also evaluated the internal consistency of the SHAPS using Cronbach's coefficient alpha $(\alpha)$.

A Principal Factor Analysis, with Promax rotation, was used to define the dimensionality of the SHAPS and to interpret the proportion of explained variance. The prior communality estimates for each SHAPS item was set to its squared multiple correlation with all other SHAPS items. To interpret a unidimensional scale, the eigenvalue of the first principal factor should exceed one (using the Kaiser-Guttmann criterion), but the reverse should be the case of all subsequent eigenvalues.

Convergent and discriminant validity was assessed by evaluating the Pearson correlations between the SHAPS total score and the pleasure/enjoyment item of the IDS- $\mathrm{C}_{30}$ (sub-item 21), QLES-Q, HRSD $_{17}$, IDS-C ${ }_{30}$, QIDS-C 16 , and MADRS ${ }_{10}$, respectively. Further, the one-way Analysis of Variance (ANOVA) was used to examine, on the SHAPS total score, those who were "severely-to-very severely depressed" ( $\geq 37$ IDS- $C_{30}$ total score) versus those who were "mild-to-moderately depressed" ( $\leq 36$ IDS- $_{30}$ total score). Also, the pleasure/enjoyment item of the IDS- $\mathrm{C}_{30}$ (sub-item 21) was categorized into three groups (those who scored 0 vs. 1 vs. 2 or 3 ) and then the one-way ANOVA was used to evaluate the relationship between the three groups of the IDS- $\mathrm{C}_{30}$ sub-item 21 and the SHAPS total score. The Ryan-Einot-GabrielWelsch Multiple Range Test (which controls the Type I experimentwise error rate) was used to examine the pairwise comparisons among the three groups of the IDS- $\mathrm{C}_{30}$ sub-item 21 .

All analyses were carried out using SAS 9.2 (SAS Institute, Inc., Cary, NC). The level of significance for all tests was set at $\alpha=.05$ (two-tailed).

\section{Results}

\section{Participant Characteristics}

The study sample included 75 males (16.3\%) and 386 females (83.7\%), with an average age of 50.2 years ( $\mathrm{SD}=10.6$; age range: 19-80 years). Participants included 282 (61.2\%)

Caucasians, 141 (30.6\%) African-Americans, 20 (4.3\%) Hispanics, and 18 (3.9\%) other (not specified). Approximately $15 \%$ of the participants were in their first episode of depression. The average length of illness was 29.5 years $(\mathrm{SD}=14.4)$, and the average duration of the current depressive episode was 9.3 years $(\mathrm{SD}=11.9)$. Participant demographic and clinical characteristics (baseline and exit) are reported in Table 2.

\section{SHAPS and Participant Characteristics}

The Pearson partial correlations (controlling for QIDS- $\mathrm{C}_{16}$ at exit) revealed no significant relationship between SHAPS total score at exit and age $(r=-0.07, p<.19)$, education $(r=-0.05$, $p<.33)$, duration of the current depressive episode $(r=-0.05, p<.32)$, and length of illness $(r=$ $0.04, p<.40$ ). The point-biserial partial correlations (controlling for QIDS- $\mathrm{C}_{16}$ at exit) also revealed no significant relationship between SHAPS total score at exit and gender $\left(r_{\mathrm{pb}}=-0.06\right.$, $p<.23)$, race $\left(r_{\mathrm{pb}}=-0.06, p<.24\right)$, and first versus recurrent episode of depression $\left(r_{\mathrm{pb}}=0.08\right.$, $p<.11)$. The results are reported in Table 3. 


\section{Internal Consistency and Scale Dimensionality}

The internal consistency (Cronbach's coefficient alpha) of the SHAPS was .91. The mean itemtotal correlation was .61 (ranging from .49 to .72 ). Table 1 summarizes the classical test theory results for the SHAPS. Cronbach's coefficient alpha for each of the depression scales (at exit) used in the current study was as follows: $\operatorname{HRSD}_{17}(\alpha=.78)$, IDS-C$_{30}(\alpha=.86)$, QIDS-C ${ }_{16}(\alpha$ $=.79)$, and MADRS $_{10}(\alpha=.86)$.

A Principal Factor Analysis, with Promax rotation, yielded a one-factor solution for the SHAPS (eigenvalues of the first two initial factors were 5.95 and 0.43 , respectively). All items loaded reasonably high (> .45) on the first principal factor, which explained $36.6 \%$ of the total variance.

\section{Convergent and Discriminant Validity}

When the pleasure/enjoyment item of the IDS- $\mathrm{C}_{30}$ (sub-item 21) was categorized into three groups (those who scored 0 vs. 1 vs. 2 or 3), the one-way ANOVA revealed a significant between-subjects effect ( 3 groups of IDS- $\mathrm{C}_{30}$ sub-item 21 ) on the SHAPS total score $(F=$ $41.59, d f=2,363, r=0.48, p<.0001)$. The means for SHAPS total scores were lower for those who scored "zero" on IDS- $\mathrm{C}_{30}$ sub-item 21 ("derives usual sense of enjoyment from pleasurable activities," $\mathrm{M}=1.98, \mathrm{SD}=3.01$ ) than those who scored "one" on IDS- $\mathrm{C}_{30}$ subitem 21 ("does not feel usual enjoyment from pleasurable activities," $\mathrm{M}=2.87, \mathrm{SD}=3.51$ ) or those who scored "two or three" on IDS-C 30 sub-item 21 ("rarely derives pleasure from any activities" or "unable to register any sense of pleasure/enjoyment from anything," $\mathrm{M}=5.96$, $\mathrm{SD}=4.46$ ). Multiple comparisons of the three coded IDS- $\mathrm{C}_{30}$ sub-item 21 groups on the SHAPS total score indicated a significant difference between those who scored "two or three" and those who scored "one" $(p<.0002)$ and between those who scored "two or three" and those who scored "zero" $(p<.0002)$, but no difference between those who scored "zero" and those who scored "one" $(p<.07)$. Further, the SHAPS total score was negatively correlated with the QLES-Q $(r=-0.65, \mathrm{p}<.0001)$.

The Pearson correlations revealed a positive linear relationship between the SHAPS total score and the total scores on the $\operatorname{HRSD}_{17}(r=.49, p<.0001)$, IDS-C $30(r=.56, p<.0001)$, QIDS$\mathrm{C}_{16}(r=.55, p<.0001)$, and MADRS $10(r=.53, p<.0001)$. The direction and magnitude of these correlation coefficients suggests that persons with greater levels of depression severity had relatively higher levels of anhedonia. Indeed, patients at exit who were "severely-to-very severely depressed" ( $\geq 37$ IDS-C 30 total score) had significantly higher mean SHAPS total scores (higher levels of anhedonia) than those who were "mild-to-moderately depressed" ( $\leq$ 36 IDS- $_{30}$ total score), $\mathrm{M}=6.65$ ( $\mathrm{SD}=4.07$ ) versus $\mathrm{M}=2.34$ ( $\left.\mathrm{SD}=3.41\right), t=9.36, p<.0001$, $\mathrm{R}^{2}=.22, r=.46$. Correlations between the SHAPS and the other measures are displayed in Table 3 .

\section{Discussion}

The current study examined the reliability and validity of the SHAPS in a large sample of 461 adult outpatients with MDD. The internal consistency of the SHAPS in this depressed outpatient sample is excellent $(\alpha=0.91)$ and is in line with previous findings in depressed and schizophrenic inpatients (e.g., Franken et al., 2007; Franz et al., 1998; Loas et al., 1997) and in depressed and non-depressed patients diagnosed with Parkinson's disease (Lemke et al., 2006). The Principal Factor Analysis indicates that the SHAPS is unidimensional, suggesting that it is limited mainly to the trait of hedonic capacity, which also accords with previous findings (Franken et al., 2007; Lemke et al., 2006; Leventhal et al., 2006). Together with results from the few existing studies, the SHAPS (English and foreign versions) seems to have substantial internal consistency and is unidimensional in the assessment of hedonic capacity 
in both patient (Franken et al., 2007; Franz et al., 1998; Lemke et al., 2006; Loas et al., 1997) and non-patient volunteer populations (Leventhal et al., 2006).

Convergent and discriminant validity were assessed by evaluating the relationship between the SHAPS total score and four separate measures of depression severity, the pleasure/enjoyment item of the IDS- $\mathrm{C}_{30}$ (sub-item 21), and a measure of Quality of Life, Enjoyment, and Satisfaction. The pattern of the SHAPS mean scores is consistent with the scoring direction on the pleasure/enjoyment item of the IDS- $\mathrm{C}_{30}$ (sub-item 21 ); that is, those who rarely or are unable to derive pleasure from activities (as coded/scored on IDS- $\mathrm{C}_{30}$ sub-item 21) also have higher levels of anhedonia or lower levels of hedonic capacity (as measured via the SHAPS). The SHAPS also correlated negatively with the QLES-Q, indicating that persons with lower levels of enjoyment and satisfaction with activities (as measured by the QLES-Q) have higher levels of anhedonia (or lower levels of hedonic capacity as measured by the SHAPS). The magnitude of the correlation between the SHAPS total score and each of the other four measures of depression is modest--and in line with previous findings that also report a modest correlation with the Beck Depression Inventory (Franken et al., 2007; Leventhal et al., 2006), HRSD (Franz et al., 1998), and MADRS (Mazzaa et al., 2009)--suggesting that hedonic capacity (as measured by the SHAPS) may tap a related but distinct construct from depression. The SHAPS is also able to discriminate between outpatients who were severely-to-very severely depressed and those who were mild-to-moderately depressed. The results of the current study in an outpatient sample of adult patients with MDD and those from previous findings (e.g., Franken et al., 2007; Franz et al., 1998; Loas et al., 1997; Leventhal et al., 2006; Mazzaa et al., 2009) suggest that the SHAPS has adequate construct validity.

Finally, in this study, we examined the relationship of the SHAPS with participant demographic and clinical characteristics. The SHAPS was not influenced by age, gender, race, education, duration of the current depressive episode, length of illness, or first versus recurrent episode of depression. This basic finding is consistent with the Franken et al. (2007) study and is in line with the supposition that the SHAPS was developed to minimize cultural, gender, and age biases in the evaluation of hedonic capacity (Snaith et al., 1995).

The current study may be tempered by a few limitations. The study evaluated the self-report SHAPS against clinician-rated measures of depression severity; it would have been preferable to validate the SHAPS against self-report measures of depression (but self-report measures of depression were not collected in the Project IMPACTS study). Also, because the evaluation of convergent validity of the SHAPS in the current study was limited in scope, the inclusion of a more comprehensive anhedonia scale or sub-scale would have strengthened the assessment of convergent validity of the SHAPS.

Despite these limitations, the current study has strengths, including a large sample of 461 adult outpatients with major depressive disorder. Further, the participants in the current study are typical outpatients with major depressive disorder who reside in the community, United States public mental health setting. The use of outpatients from the public health care system, thus, provides a greater degree of generalizability of our findings.

\section{Conclusion}

In conclusion, this is the first study to our knowledge to show that the original (English) version of the SHAPS (Snaith et al., 1995) has excellent internal consistency, with construct validity, and is unidimensional in assessing hedonic capacity among adult outpatients with major depressive disorder. The SHAPS possesses excellent psychometric properties, was not influenced by participant demographic and clinical characteristics, and appears appropriate for use in both clinical and research settings. 


\section{Acknowledgments}

This work is supported by R01 MH-164062-01A1, Computerized Decision Support System for Depression (CDSSD), awarded through the National Institute of Mental Health, Madhukar H. Trivedi, M.D., Principal Investigator.

\section{References}

American Psychiatric Association. Diagnostic and Statistical Manual of Mental Disorders. 4. Washington DC: American Psychiatric Press; 2000. Text Revision

Endicott J, Nee J, Harrison W, Blumenthal R. Quality of Life Enjoyment and Satisfaction Questionnaire: a new measure. Psychopharmacology Bulletin 1993;29:321-326. [PubMed: 8290681]

First, MB.; Spitzer, RL.; Gibbon, M.; Williams, JBW. Structured Clinical Interview for DSM-IVAxis I Disorders, Clinician Version (SCID-1-CV). New York, NY: Biometrics Research Department, New York State Psychiatric Institute, Department of Psychiatry, Columbia University; 1995.

Franken I, Rassin E, Muris P. The assessment of anhedonia in clinical and non-clinical populations: Further validation of the Snaith-Hamilton Pleasure Scale (SHAPS). Journal of Affective Disorders 2007;99:83-89. [PubMed: 16996138]

Franz M, Lemke MR, Meyer T, Ulferts J, Puhl P, Snaith RP. German version of the Snaith-HamiltonPleasure Scale (SHAPS-D). Anhedonia in schizophrenic and depressive patients. Fortschr Neurol Psychiatr 1998;66:407-413. [PubMed: 9782420]

Galinowski A, Lehert P. Structural validity of MADRS during antidepressant treatment. International Clinical Psychopharmacology 1995;10:157-161. [PubMed: 8675968]

Hamilton M. A rating scale for depression. Journal of Neurology Neurosurgery and Psychiatry 1960;12:56-62.

Khan A, Khan SR, Shankles EB, Polissar NL. Relative sensitivity of the Montgomery-Asberg Depression Rating Scale, the Hamilton Depression Rating Scale, and the Clinical Global Impressions rating scale in antidepressant clinical trials. International Clinical Psychopharmacology 2002;17:281-285. [PubMed: 12409681]

Lemke MR, Brecht HM, Koester J, Reichmann H. Effects of the dopamine agonist pramipexole on depression, anhedonia and motor functioning in Parkinson's disease. Journal of the Neurological Sciences 2006;249:266-270. [PubMed: 16814808]

Leventhal AM, Chasson GS, Tapia E, Miller EK, Pettit JW. Measuring hedonic capacity in depression: a psychometric analysis of three anhedonia scales. Journal of Clinical Psychology 2006;62:15451558. [PubMed: 17019674]

Loas G, Dubal S, Perot P, Tirel F, Nowaczkowski P, Pierson A. Validation of the French version of the Snaith-Hamilton Pleasure Scale (SHAPS, Snaith et al. 1995). Determination of the statistical parameters in 208 normal subjects and 103 hospitalized patients presenting with depression or schizophrenia. Encephale 1997;23:454-458. [PubMed: 9488929]

Mazza M, Squillacioti MR, Pecora RD, Janiri L, Bria P. Effect of aripiprazole on self-reported anhedonia in bipolar depressed patients. Psychiatry Research 2009;165:193-196. [PubMed: 18973955]

Montgomery SA, Äsberg M. A new depression scale designed to be sensitive to change. British Journal of Psychiatry 1979;134:382-389. [PubMed: 444788]

Rush AJ, Gullion CM, Basco MR, Jarrett RB, Trivedi MH. The Inventory of Depressive Symptomatology (IDS): psychometric properties. Psychological Medicine 1996;26:477-486. [PubMed: 8733206]

Rush AJ, Trivedi MH, Ibrahim HM, Carmody TJ, Arnow B, Klein DN, Markowitz JC, Ninan PT, Kornstein S, Manber R, Thase ME, Kocsis JH, Keller MB. The 16-Item Quick Inventory of Depressive Symptomatology (QIDS), clinician rating (QIDS-C), and self-report (QIDS-SR): a psychometric evaluation in patients with chronic major depression. Biological Psychiatry 2003;54:573-583. [PubMed: 12946886]

SAS. Cary, NC: Statistical Analysis System Institute;

Schwab JJ, Bialow MR, Holzer CE. A comparison of two rating scales for depression. Journal of Clinical Psychology 1967;23:94-96. [PubMed: 6031042]

Snaith RP. Anhedonia: a neglected symptom of psychopathology. Psychological Medicine 1993;23:957966. [PubMed: 8134519] 
Snaith RP, Hamilton M, Morley S, Humayan A, Hargreaves D, Trigwell P. A scale for the assessment of hedonic tone the Snaith-Hamilton Pleasure Scale. British Journal of Psychiatry 1995;167:99-103. [PubMed: 7551619]

Trivedi MH, Rush AJ, Ibrahim HM, Carmody TJ, Biggs MM, Suppes T, Crismon ML, Shores-Wilson K, Toprac MG, Dennehy EB, Witte B, Kashner TM. The Inventory of Depressive Symptomatology, Clinician Rating (IDS-C) and Self-Report (IDS-SR), and the Quick Inventory of Depressive Symptomatology, Clinician Rating (QIDS-C) and Self-Report (QIDS-SR) in public sector patients with mood disorders: a psychometric evaluation. Psychological Medicine 2004;34:73-82. [PubMed: 14971628]

Trivedi MH, Claassen CA, Grannemann BD, Kashner TM, Carmody TJ, Daly E, Kern JK. Assessing Physicians' Use of Treatment Algorithms: Project IMPACTS Study Design and Rationale. Contemporary Clinical Trials 2007;28:192-212. [PubMed: 16997636]

World Health Organization. Clinical Descriptors and Diagnostic Guidelines. Geneva: World Health Organization; 1992. The ICD-10 Classification of Mental and Behavioural Disorders. 


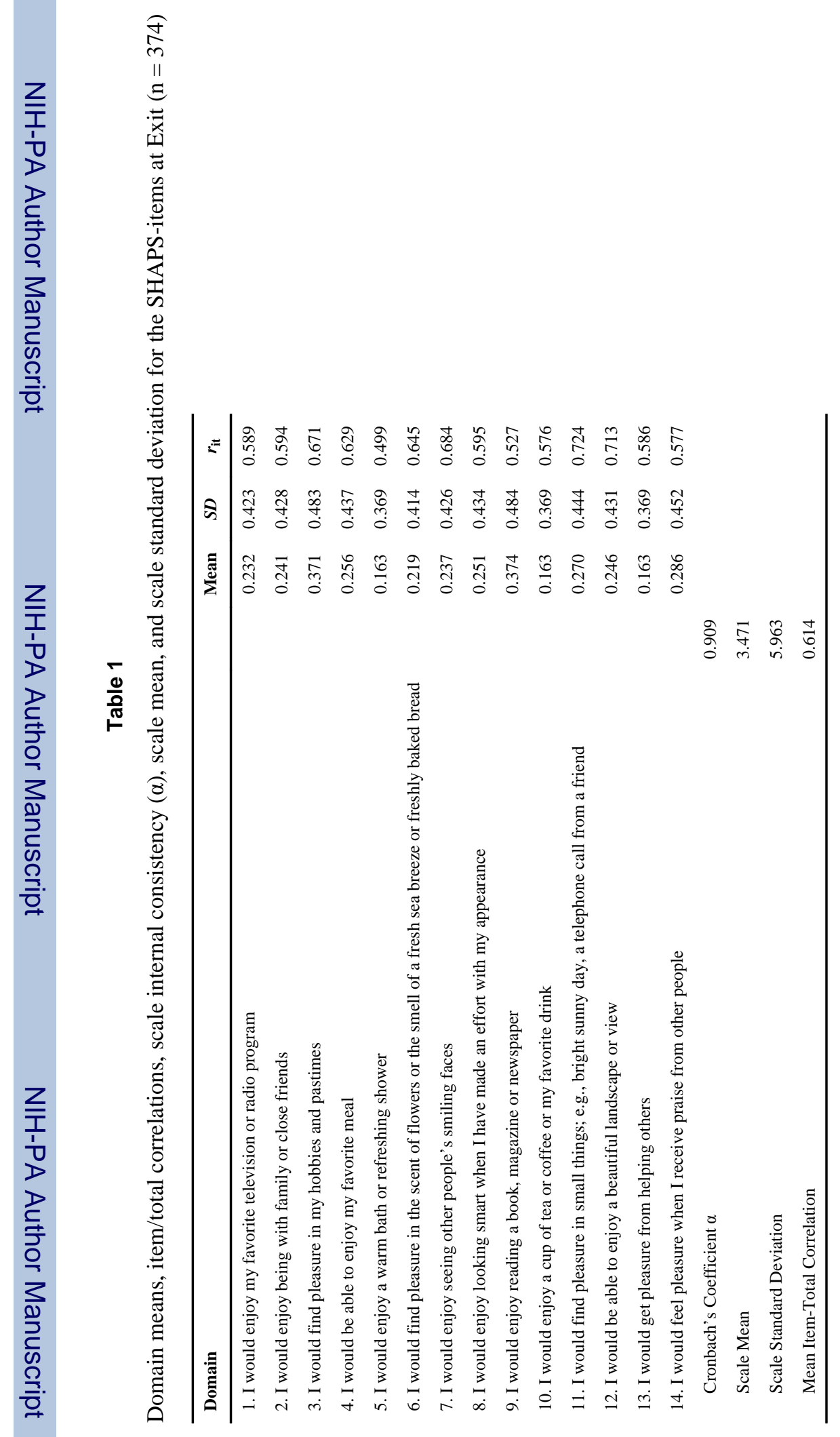

Int Clin Psychopharmacol. Author manuscript; available in PMC 2011 November 1. 
Table 2

Participant Characteristics

\begin{tabular}{|c|c|}
\hline Participant Characteristic & Patient Sample $(N=461)$ \\
\hline \multicolumn{2}{|l|}{ Demographics } \\
\hline Age in years, $\mathrm{M}(\mathrm{SD})$ & $50.2(10.6)$ \\
\hline Education in years, $\mathrm{M}(\mathrm{SD})$ & $12.5(2.6)$ \\
\hline \multicolumn{2}{|l|}{ Race, $\mathrm{N}(\%)$} \\
\hline Caucasian & $282(61.2)$ \\
\hline African American & $141(30.6)$ \\
\hline Hispanic & $20(4.3)$ \\
\hline Other (not specified) & $18(3.9)$ \\
\hline \multicolumn{2}{|l|}{ Gender, N (\%) } \\
\hline Female & $386(83.7)$ \\
\hline Male & $75(16.3)$ \\
\hline Marital Status: Married, N (\%) & $112(24.3)$ \\
\hline Employment Status: Employed, N (\%) & $27(5.9)$ \\
\hline \multicolumn{2}{|l|}{ Clinical Characteristics } \\
\hline Psychotic Features, N (\%) & $104(22.5)$ \\
\hline Anxiety, N (\%) & $257(55.7)$ \\
\hline Substance Use, N (\%) & $195(42.2)$ \\
\hline Number of GMCs, M (SD) & $12.4(6.8)$ \\
\hline First MDD Episode, N (\%) & $71(15.5)$ \\
\hline Current MDD Episode Length in years, M (SD) & $9.3(11.9)$ \\
\hline Age of MDD Onset in years, M (SD) & $20.8(13.9)$ \\
\hline Length of Illness in years, $M(\mathrm{SD})$ & $29.5(14.4)$ \\
\hline SHAPS total at baseline, $M(\mathrm{SD})$ & $4.0(3.3)$ \\
\hline SHAPS total at exit, M (SD) & $3.4(4.1)$ \\
\hline $\mathrm{HRSD}_{17}$ total at baseline, $\mathrm{M}(\mathrm{SD})$ & $21.7(4.6)$ \\
\hline $\mathrm{HRSD}_{17}$ total at exit, $\mathrm{M}(\mathrm{SD})$ & $15.5(6.5)$ \\
\hline IDS- $\mathrm{C}_{30}$ at baseline, $\mathrm{M}(\mathrm{SD})$ & $37.7(8.4)$ \\
\hline IDS- $\mathrm{C}_{30}$ at exit, $\mathrm{M}(\mathrm{SD})$ & $28.2(12.9)$ \\
\hline IDS- $\mathrm{C}_{30}$ sub-item 21 at baseline, $\mathrm{M}$ (SD) & $1.1(0.82)$ \\
\hline IDS- $C_{30}$ sub-item 21 at exit, M (SD) & $0.89(0.86)$ \\
\hline QIDS- $\mathrm{C}_{16}$ at baseline, $\mathrm{M}(\mathrm{SD})$ & $15.7(3.5)$ \\
\hline QIDS- $_{16}$ at exit, $\mathrm{M}$ (SD) & $11.1(5.2)$ \\
\hline MADRS $_{10}$ at baseline, $\mathrm{M}$ (SD) & $27.9(7.3)$ \\
\hline $\mathrm{MADRS}_{10}$ at exit, M (SD) & $19.4(9.9)$ \\
\hline QLES-Q at baseline, M (SD) & $48.1(11.8)$ \\
\hline QLES-Q at exit, M (SD) & $53.3(14.7)$ \\
\hline
\end{tabular}

Note: $\mathrm{M}=$ Mean; $\mathrm{SD}=$ Standard Deviation; $\mathrm{N}=$ Sample Size.

IDS-C 30 sub-item $21=$ the pleasure/enjoyment item of the IDS-C 30 . 
GMC $=$ General Medical Conditions. 


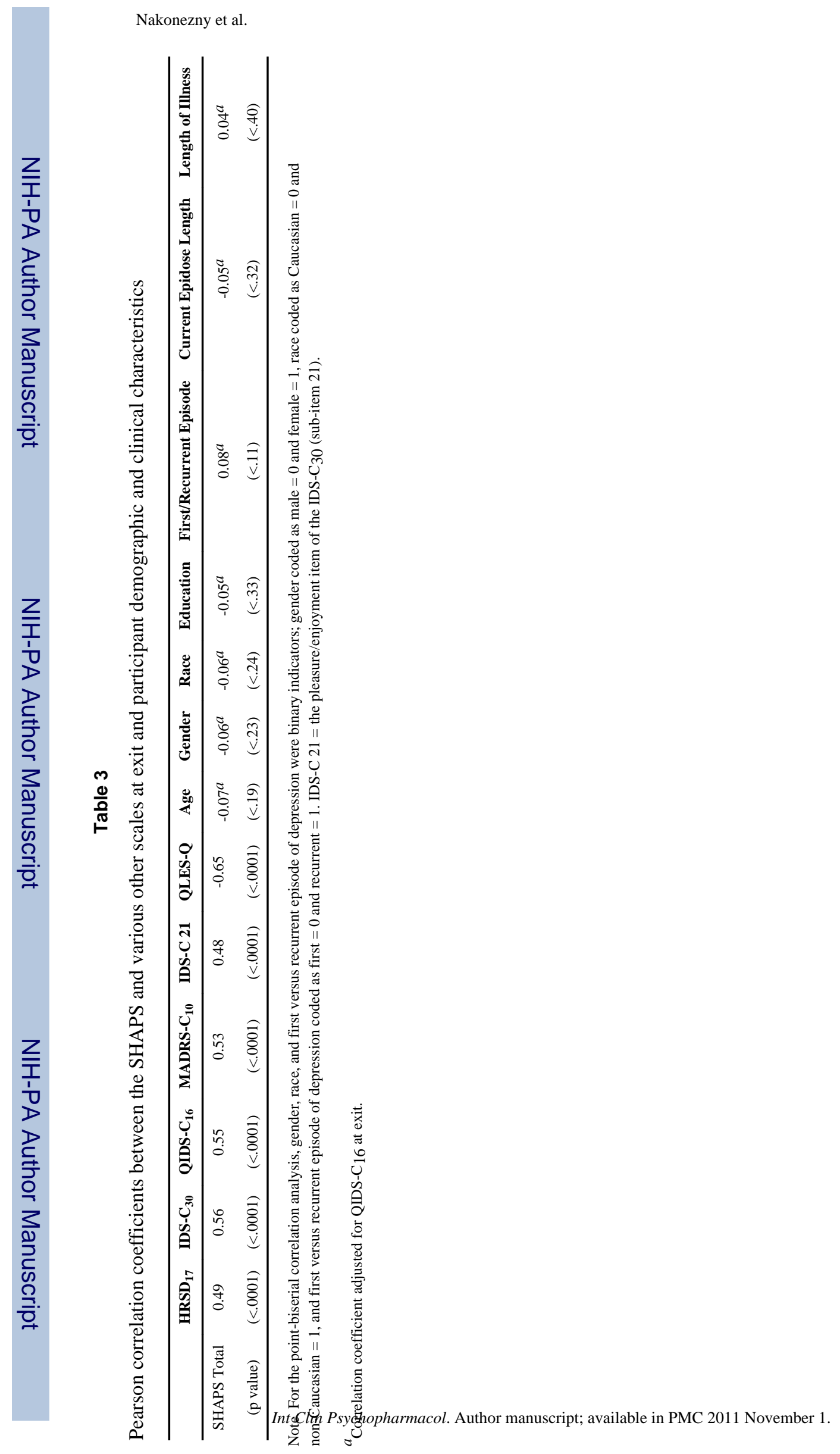

Page 12 